\title{
INTEGRATED TESTING OF POLYMER MEMS MATERIAL PROPERTIES
}

\author{
D. Sameoto ${ }^{1}$, C. Plesa ${ }^{1}$, and M. Parameswaran ${ }^{1}$ \\ ${ }^{1}$ Institute of Micromachine and Microfabrication Research, Simon Fraser University, Burnaby, BC, Canada
}

\begin{abstract}
We present a novel method for rapidly testing the material properties and fabrication qualities of polymer MEMS structures that uses an external vibration source and an all optical measurement system. A combination of static and dynamic test structures are designed and fabricated in SU-8 to test the basic performance of the system, and automatic measurements of device dimensions are used to correct for non-ideal fabrication effects. A piezoelectric actuator directly connected to the substrate excites all compliant structures in-plane simultaneously, and the resonance response is measured optically. The Young's modulus of SU-8 is found to be $3.88 \pm 0.20 \mathrm{GPa}$ using this system.
\end{abstract}

\section{INTRODUCTION}

Polymers are becoming increasingly popular for MEMS applications due to their wide range of material properties, low temperature processing and generally low costs. Polymers have been used for rapid MEMS prototyping [1], fabrication of microfluidics [2], and for thermal isolation of sensors [3]. More recently, novel composite structures have been developed that add electrical functionality to polymer MEMS devices [4] for sensing or actuation purposes. In order to achieve good yields and determine material properties for new composites or processing parameters, an integrated system for rapidly testing polymer MEMS properties is required.

The evaluation of material properties in MEMS is extremely important for determining performance of fabricated devices, expected failure modes, and long term reliability. Non-integrated testing may be performed by removing components and performing macroscale type tests for stress-strain relations, fracture strength etc., but they are not practical for rapid material property extraction. Previous publications have identified three major types of integrated test structures: M-test, strain gauge and resonance [5]. Of these three, only strain gauges and resonance structures are appropriate for the thin and thick structures that can be fabricated with polymer MEMS [5], A standard test die was designed for this work that includes many of these test structures (Figure 1).

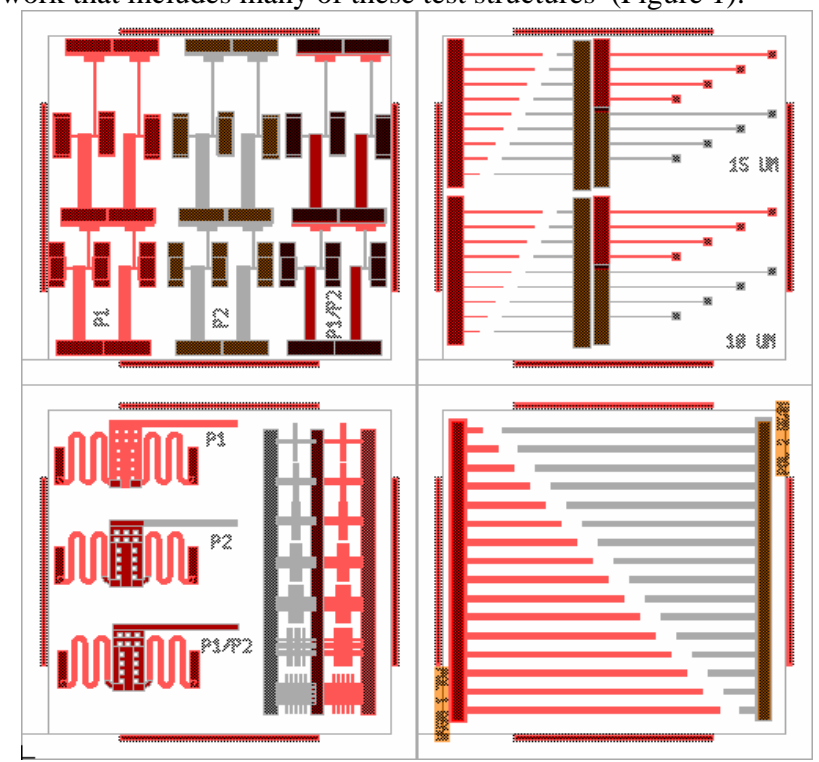

Figure 1: L-Edit layout of a 1x1 cm polymer MEMS test die.
The resonance of MEMS structures has often been used to determine Young's modulus [6], but electrostatic actuation for high frequency excitation is not possible with electrically insulating polymers. To overcome this problem, an external excitation source is used on the whole die or wafer at a single time, while in-plane dynamic response is measured optically. In-plane resonance is easily observed with a standard microscope, and is theoretically independent of device thickness. Fixed-free cantilevers can provide values for Young's modulus, while fixedfixed beams can be used to evaluate residual stress. Other static structures like strain gauges [7], or cantilevers attached to out-ofplane pop-up structures [8] may be used to directly observe residual strain or curvature, but cannot be used directly to find stress or stress gradients unless the Young's modulus is known. When combined with data from the resonance structures, these structures can be used to extract other material properties. However, the focus of this work is how to best measure polymer material properties through frequency response.

\section{FABRICATION}

Fabrication of the polymer MEMS test die follows a very similar method to that described in [1]. Rather than a polystyrene sacrificial layer used in previous work [1], a polyimide based sacrificial layer (Prolift [9]), is used. Prolift dissolves rapidly in TMAH-based developers, can be processed above $200{ }^{\circ} \mathrm{C}$ while remaining soluble, and is unaffected by most organic solvents. Therefore, it is an excellent sacrificial layer for many spin-coated polymers. A thick layer of Shipley 1827 photoresist is spun-coat on the Prolift and patterned with an underexposure, so as not to remove Prolift during the development step. A second, blanket underexposure can also be used through a bare Mylar sheet to roughen the remaining photoresist surface significantly (Figure 2).

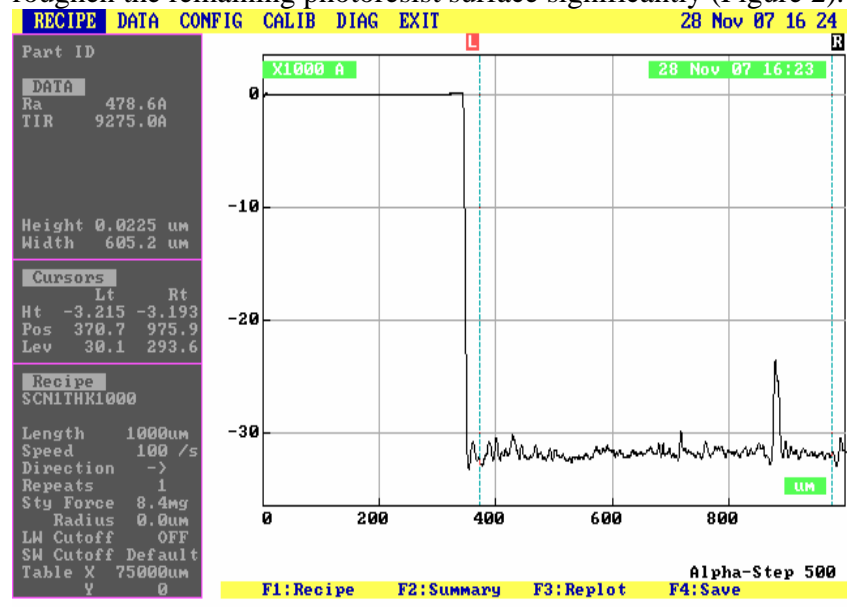

Figure 2: Profilometer data of an underexposed anchor in photoresist defined using a Mylar mask. Optical imperfections in the Mylar produce a very rough photoresist surface.

An $\mathrm{O}_{2}$ RIE step transfers the photoresist topology to the Prolift and results in a very rough sacrificial layer that minimizes stiction after a wet release while also significantly increases the visibility of transparent polymers. With thicker polymer MEMS structures, fabricated dimensions must be experimentally measured to ensure accurate measurements of material properties from strain 
gauges and resonant structures. SU-8 is used as a structural layer in this work because it is a common polymer used in MEMS and material properties can be compared with previously published results. Two different structural thicknesses are defined on each test die, either 18 or $38 \mu \mathrm{m}$ thick in this work, to determine the effects of the resonating test structures with different aspect ratios. The basic process steps are shown in Figure 3.
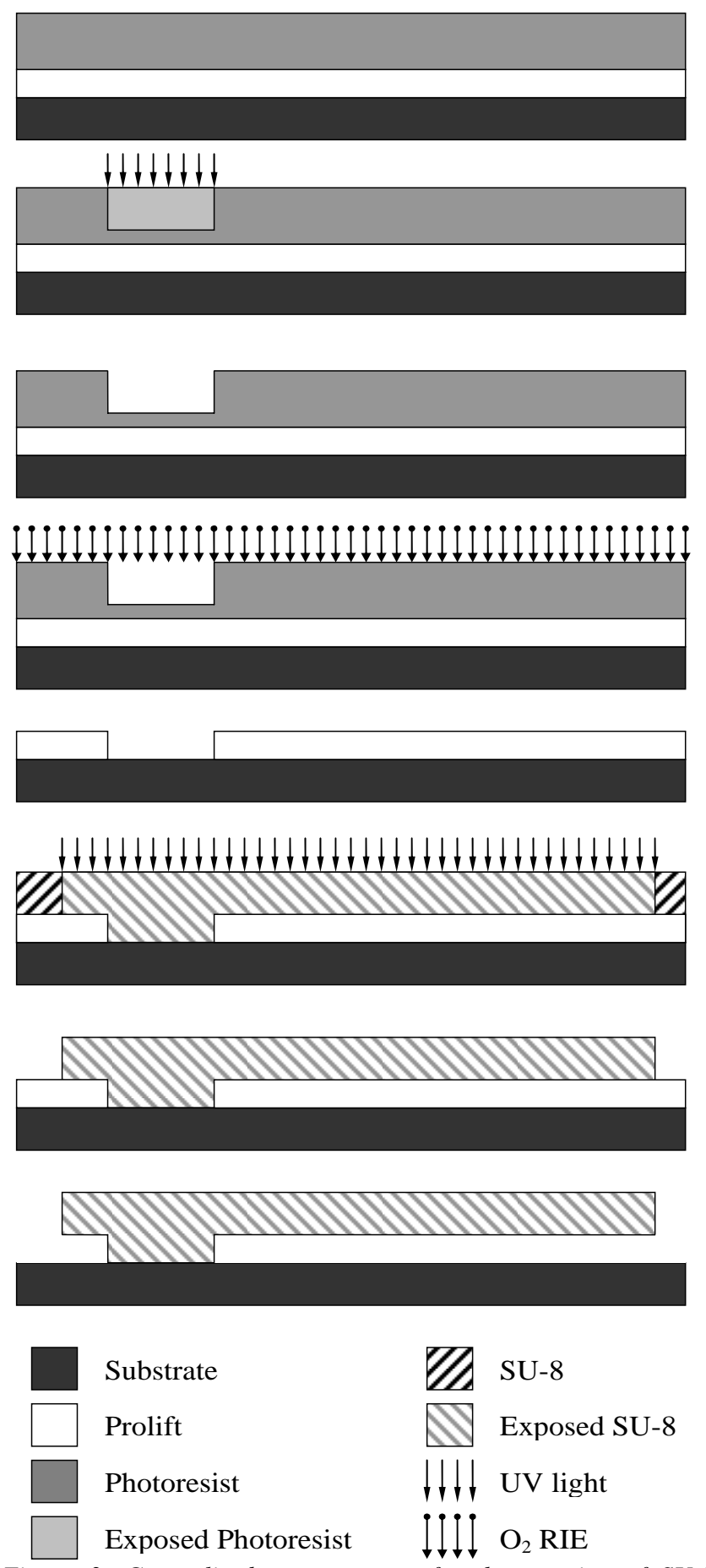

$\begin{array}{lll}\text { Substrate } & \\ \text { Prolift } & & \text { SU-8 } \\ \text { Photoresist } & & \\ \text { Exposed Photoresist } & \downarrow \downarrow \downarrow \downarrow & \mathrm{O}_{2} \text { RIE }\end{array}$

Figure 3: Generalized process steps for the creation of SU-8 MEMS cantilevers for dynamic testing.

\section{TEST SETUP}

Individual test die, or wafer sections are placed under a microscope after release and examined using either a Moticam 1300 (resolution 1024x768), or a BigCatch EM-500M eyepiece camera (resolution 2592x1944). The higher resolution of the BigCatch camera is offset by a much lower refresh rate, making it better suited for static images or slower frequency sweeps. A National Instruments PCI-5401 function generator card is used to step a sinusoidal signal through a frequency range. This signal is input to a Trek model PZD-700 high voltage amplifier capable of outputting $\pm 700 \mathrm{~V}$ to a piezoelectric actuator. This piezoelectric actuator excites the substrate in-plane for dynamic characterization of polymers with the same microscope setup used for static measurements (Figure 4). Images are captured corresponding to each input frequency, with each step taking approximately 4 seconds.
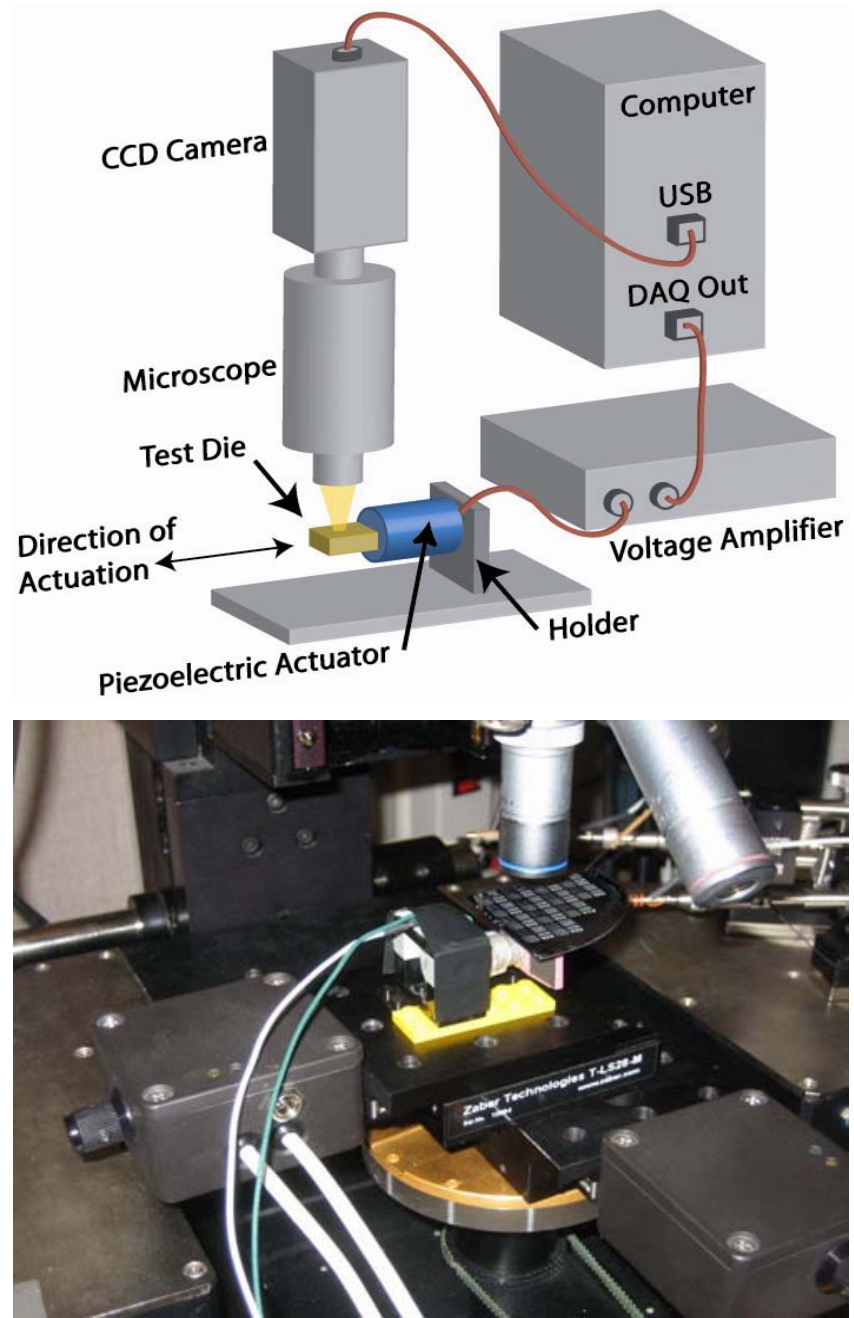

Figure 4: Schematic of test setup and image of a quarter wafer in position for testing. The existing test system can work on a few die at once, but should be able to eventually measure whole wafers automatically.

The substrate motion is lower than the optical threshold of the system and can be ignored while taking measurements. Polymer structures typically have low values for Young's modulus $(<10$ $\mathrm{GPa}$ ), and can resonate at frequencies below the resonance of the piezoelectric crystal (Figure 5). After all the images are captured during the frequency sweep, National Instruments Vision Builder for Automated Inspection is used to determine device dimensions and resonance points. Edge detection in regions of interest allows 
the response of multiple structures within the field of view to be measured and evaluated. Matlab ${ }^{\circledR}$ is then used to calculate material properties from the dimensions and resonance frequencies of multiple structures, and average values and standard deviation for Young's modulus are found.
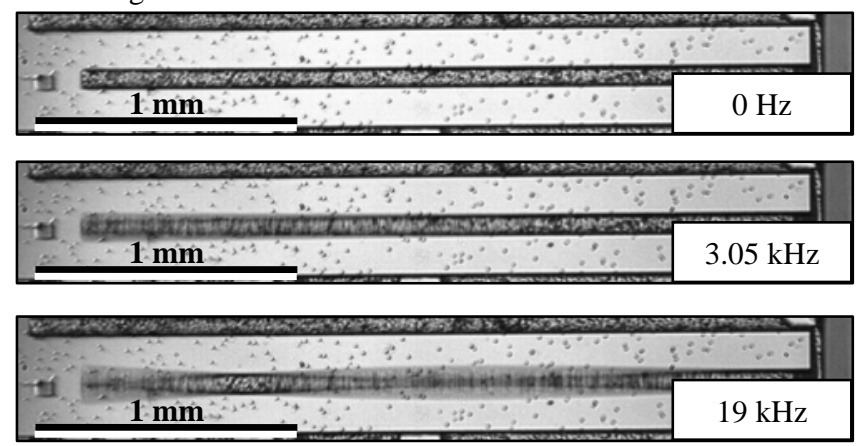

Figure 5: Dynamic response of a long, low aspect ratio ( 1:2) SU8 cantilever at different input frequencies. Two resonance modes are observed below the resonance frequency of the piezoelectric crystal $(25 \mathrm{kHz})$.

\section{THEORY}

Basic beam theory allows Young's modulus to be found from the undamped resonance frequency of a cantilever beam by the following relationship assuming a rectangular cross section:

$$
f_{n}=\frac{C_{n}}{2 \pi} \sqrt{\frac{E w^{2}}{12 \rho L^{4}}}
$$

Where $\rho$ is the density of the material, $L$ is length, $E$ is Young's modulus and $w$ is beam width. In this case, $C_{n}$ is the coefficient corresponding to the resonant mode of the beam (3.52 for the first mode of a cantilever). Matlab ${ }^{\circledR}$ is used to determine mechanical properties based on the measured device geometries and resonant frequencies. The density of SU-8 is assumed to be $1200 \mathrm{~kg} / \mathrm{m}^{3}$ for these calculations. For new polymers or composites tested in the future, data may be calibrated based on measured densities. The out-of-plane structures used for curvature measurement can also be used to directly observe the sidewall angle to provide correction factors for the equations used for resonance based on trapezoidal cross-sections (Figure 6). The average sidewall angle was measured to be approximately $85^{\circ}$.

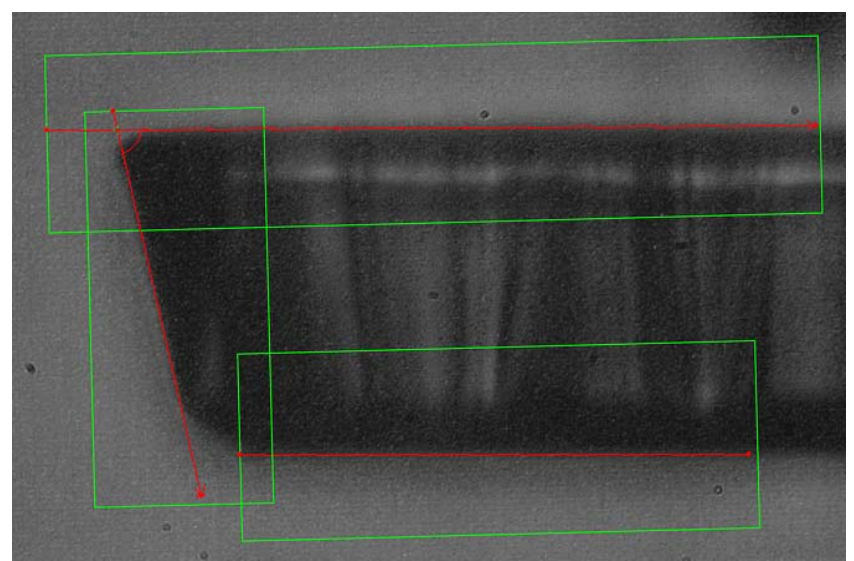

Figure 6: Negative sidewalls in SU-8 structures due to exposure through a Mylar emulsion mask.

The corrected natural frequency for a trapezoidal beam resonating in-plane is found by the following equation, where $w_{b}$ is the bottom width, and $w_{t}$ is the top width.

$$
f_{n}=\frac{C_{n}}{2 \pi} \sqrt{\frac{E\left(w_{b}^{3}+w_{b}^{2} w_{t}+w_{b} w_{t}^{2}+w_{t}^{3}\right)}{12 \rho L^{4}\left(3 w_{b}+w_{t}\right)}}
$$

\section{RESULTS}

Measurements were made on over 90 different SU-8 resonant structures across the test wafer. The majority of narrow compliant SU-8 structures fabricated in early experiments (upper right quadrant of the test die), had feature swelling due to contact problems with the flexible Mylar masks. This swelling increased the natural frequency of all structures. In addition, many of the smaller structures with swollen features demonstrated negative curvature, due to lower exposure dose per area [10], and could not vibrate freely. The swelling of fixed-fixed beams meant that nearly all of the structures had resonance frequencies above that of the piezoelectric actuator, making measurements of these structures unreliable. Wider and thinner structures demonstrated the best fabrication yields and were also the least sensitive to errors in measurements of sidewall angle (Figure 7) and beam width.

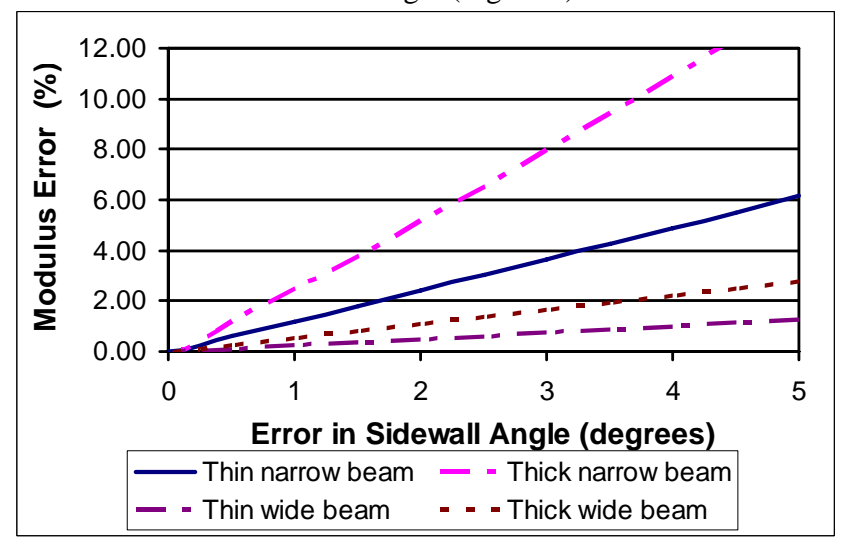

Figure 7: Theoretical error introduced into measurements of Young's modulus from sidewall angle for different beam geometries as fabricated on the test die.

The measured quality factor of tested cantilevers was greater than 30 in air, indicating that the damping introduces less than $1 \%$ error. Multiple tests on resonating structures also indicate that the material properties of SU-8 remain stable and the optical measurements are repeatable (Figure 8).

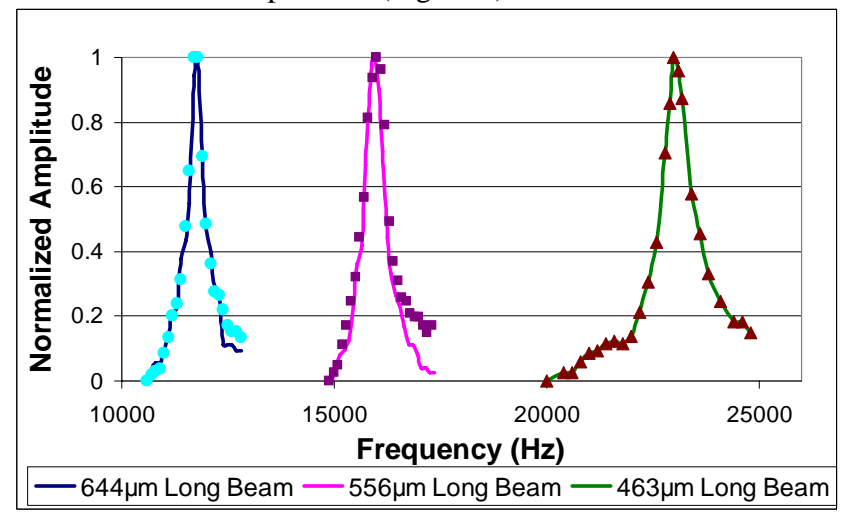

Figure 8: Resonance peaks of three narrow beams, $38 \mu \mathrm{m}$ thick on a single die measured 1 week (solid line), and 60 days (dots), after fabrication. Little change in $Q$ factor or resonance frequency of SU-8 cantilevers is observed. 
The full analysis of all beams results in a range of measured Young's modulus between 3.5 and $4.3 \mathrm{GPa}$. The majority of structures however, clustered around an average Young's modulus of $3.88 \pm 0.20 \mathrm{GPa}$ which agrees well with previously published values [11]. The average Young's modulus and standard deviation of differently designed cantilever geometries is shown below in Figure 9. Thinner, wider cantilevers demonstrate the lowest range of measured Young's modulus.

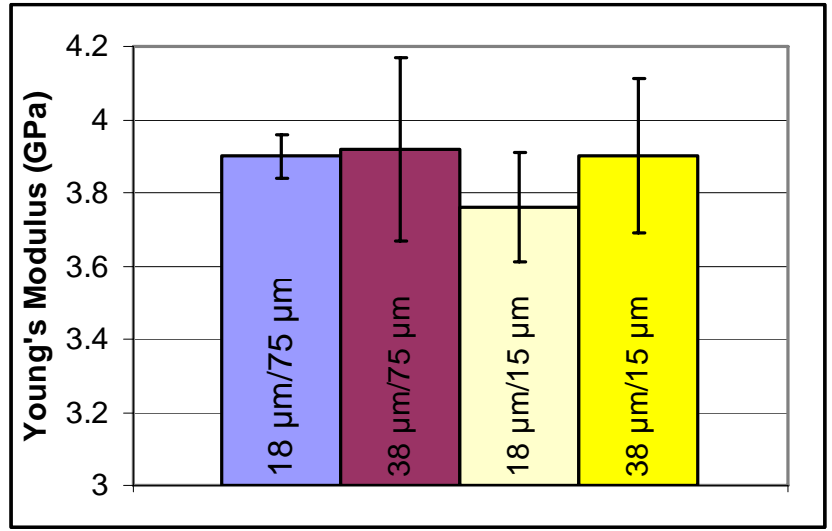

Figure 9: Measured Young's modulus for SU-8 from resonance of different thicknesses and designed widths of SU-8 cantilevers post exposure baked at $120^{\circ} \mathrm{C}$ for 30 minutes.

Given that the thinnest, longest structures showed greatest reliability, different analysis techniques were tested to determine a minimum number of tests to provide accurate results. A series of curve fits were completed on the $18 \mu \mathrm{m}$ thick, $75 \mu \mathrm{m}$ nominal width cantilevers with non-linear least squares fit from the Matlab ${ }^{\circledR}$ curve fitting toolbox (Figure 10). On average, the results agreed with the full analysis to within $0.06 \mathrm{GPa}$, indicating that acceptable extraction of Young's modulus may be achieved with as little as five different resonance measurements on the low aspect ratio beams.
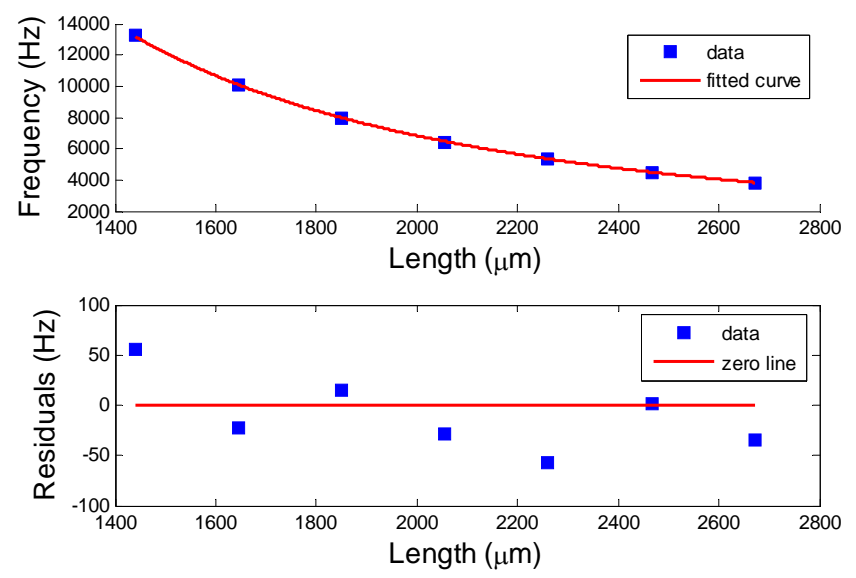

Figure 10: Least squares fit of resonance data from a set of low aspect ratio $S U-8$ cantilevers on a single die.

\section{CONCLUSIONS}

A novel test system for integrated testing of polymer MEMS has been developed that overcomes challenges with respect to actuating insulating structures and observing transparent polymers without the addition of extra materials. It is to the authors' knowledge, the first test mechanism to actuate MEMS on multiple die simultaneously and to use resonance of low aspect ratio structures in-plane to extract material properties. This system is designed to account for fabrication problems which may arise during the creation of polymer structures through lithography, direct molding or dry etching. The in-plane actuation of full die or quarter wafers using a piezoelectric actuator allows multiple structures to be tested within an optical field of view which reduces analysis times. Analysis of failure modes and data obtained from different structures indicates that low aspect ratio structures are more likely to provide acceptable data from in-plane excitation due to the more accurate measurements of structure width and smaller effect of non-vertical sidewalls. Future improvements to this system will include the introduction of piezoelectric actuators with higher resonant frequencies and lower operating voltage, so that excitation of cantilevers and fixed-fixed bars over a larger frequency range and at different frequency modes may be completed.

\section{ACKNOWLEDGEMENTS}

We would like to thank the Natural Sciences and Engineering Research Council of Canada for financial support of this work.

\section{REFERENCES}

[1] D. Sameoto, S. H. Tsang, and M. Parameswaran, "Polymer MEMS processing for multi-user applications," Sensors and Actuators A, vol. 134, pp. 457-464, 2007.

[2] S. Metz, S. Jiguet, A. Bertsch, and P. Renaud, "Polyimide and SU-8 microfluidic devices manufactured by heatdepolymerizable sacrificial material technique," Lab on a Chip, vol. 4, pp. 114-120, 2004.

[3] S. H. Tsang, A. H. Ma, K. S. Karim, M. Parameswaran, and A. M. Leung, "Monolithically fabricated polymer MEMS 3axis thermal accelerometers designed for automated wirebonder assembly," presented at IEEE MEMS 2008, Tucson, AZ, pp. 880-883.

[4] J. M. Engel, N. Chen, K. Ryu, S. Pandya, C. Tucker, Y. Yang, and C. Lie, "Multi-level embedment of conductive and nonconductive PDMS for all-elastomer MEMS," Solid State Sensors, Actuators and Microsystems Workshop (Hilton Head, SC, June 2006), pp. 316-319.

[5] V. T. Srikar and S. M. Spearing, "A critical review of microscale mechanical testing methods used in the design of microelectromechanical systems," Experimental Mechanics, vol. 43, pp. 238-247, 2003.

[6] K. E. Petersen and C. R. Guarnieri, "Young's modulus measurements of thin films using micromechanics," Journal of Applied Physics, vol. 50, pp. 6761-6766, 1979.

[7] L. Lin, A. P. Pisano, and R. T. Howe, "A micro strain gauge with mechanical amplifier," Journal of Microelectromechanical Systems, vol. 6, pp. 313-321, 1997.

[8] S.-H. Tsang, D. Sameoto, I. G. Foulds, R. W. Johnstone, and M. Parameswaran, "Automated assembly of hingeless $90^{\circ}$ out-of-plane microstructures," J. Micromech. Microeng., vol. 17, pp. 1314-1325, 2007.

[9] http://www.brewerscience.com/?id=134\#27, "Prolift Data Sheet," Brewer Science, 2008.

[10] D. Sameoto, S. H. Tsang, I. G. Foulds, S.-W. Lee, and M. Parameswaran, "Control of the out-of-plane curvature in SU-8 compliant microstructures by exposure dose and baking times," Journal of Micromechanics and Microengineering, vol. 17, pp. 1093-1098, 2007.

[11] H. Lorenz, M. Despont, N. Fahrni, N. LaBianca, and P. Renaud, "SU-8: a low-cost negative resist for MEMS," J. Micromech. Microeng., vol. 7, pp. 121-124, 1997. 